\title{
Michael Wörrle
}

\section{Ermandyberis von Limyra, ein prominenter Bürger aus der Chora*}

Ermandyberis von Limyra lebte um die Wende vom 1. zum 2. Jahrhundert n. Chr. ${ }^{1}$. Wir kennen ihn seit 1997 aus der Inschrift, die auf der Basis seiner postumen Ehrenstatue steht ${ }^{2}$ :

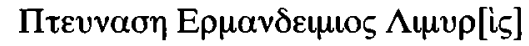

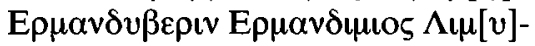

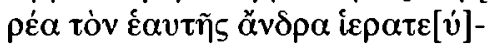

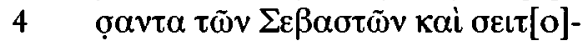

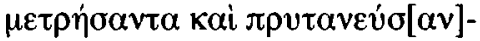

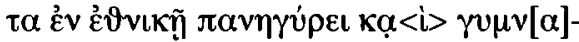

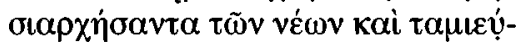

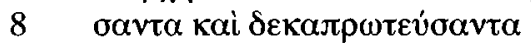

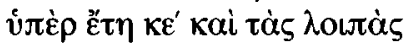

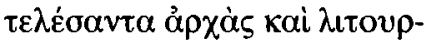

12

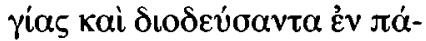

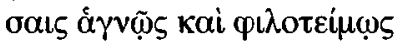

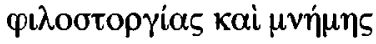

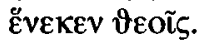

\footnotetext{
* Der folgende Beitrag soll den vorangehenden Surveybericht von Th. Marksteiner mit einer epigraphischen Fallstudie ergänzen und einen Ansatz weiterführender historischer Erwägungen aufzeigen; er kann nicht ohne Dank für die Einladung zur Teilnahme an dem BondaUnternehmen und manchen guten Rat an A. Konecny und Th. Marksteiner beginnen. Die Vortragsform ist beibehalten, in den Anmerkungen die wichtigste Dokumentation hinzugefügt.

1 Die Datierung hängt an der Dekaprotie des Ermandyberis, vgl. u. Anm. 16.

2 Die Basis (Abb. 1) steht, tief im Steinversturz steckend, in den Siedlungsruinen von Bonda (vgl. hierzu Marksteiner, o. S. 280 f. und Karte Abb. 1 auf S. 272), wo ca. 50 m nördlich oberhalb der noch heute benützten Zisterne (am Weg ca. $100 \mathrm{~m}$ westlich großer Ziegenställe) geräumige und besser ausgestattete Gebäude deren zentralen Bereich anzudeuten scheinen. Sie ist nur teilweise sichtbar und kann deshalb nicht vollständig beschrieben werden. Ihr $6,5 \mathrm{~cm}$ starkes Oberprofil endet auf der rechten Nebenseite in $68 \mathrm{~cm}$ Tiefe, was Einbindung in einen architektonischen Verband vermuten läßt, auf der Oberfläche sind Standspuren
} 
Der Vater des Ermandyberis hieß Ermandimis, und es ist kein Kunststück, beide $\mathrm{Namen}$ als ungriechisch zu erkennen. Sie gehören zum epichorisch-lykischen $\mathrm{Na}$ mengut: Der erste Bestandteil, in dem sie übereinstimmen, evoziert die Erinnerung an den luwischen Mondgott $\mathrm{Arma}^{3}$. Die Frau unseres Ermandyberis hieß Pteunase, ein Name, der mir so noch nicht begegnet, aber ohne Frage auch seinerseits der einheimischen Onomastik zuzurechnen ist $t^{4}$. Auch Pteunases Vater hieß Ermandimis, schrieb sich aber, anders als eine Zeile später der Vater des Ermandyberis, mit „ei“: Ermandeimis. Vielleicht sollten so die beiden Väter als homonyme, aber verschiedene Personen kenntlich gemacht werden, vielleicht - meines Erachtens wahrscheinlicher - handelt es sich aber nur um eine orthographische Inkonsequenz, die sachlich bedeutungslos ist. Im letzteren Fall könnten die Eheleute Geschwister sein, am ehesten solche von verschiedenen Müttern. Ehen zwischen Cousins und Cousinen waren im kaiserzeitlichen Lykien gerade auch in Elitefamilien wie den Licinnii von Oinoanda und den Arruntii von Xanthos gängige Pra$\mathrm{xis}^{5}$, wie oft oder wie selten sich Geschwister heirateten, kann ich nicht sagen, eine demographisch aussagefähige Quelle, wie sie für Teile des kaiserzeitlichen Ägypten die Zensusdeklarationen darstellen, steht für Lykien nicht zur Verfügung ${ }^{6}$. Verwandt waren Ermandyberis, dessen Name in seinem engeren Umfeld noch zweimal belegt ist ${ }^{7}$, und Pteunase wohl in jedem Fall, und dann wird man der Familie wenigstens in der Namenwahl traditionalistische Ambitionen attestieren, ohne doch in der Lage zu sein, dies sozial- und mentalitätsgeschichtlich befriedi-

ciner Bronzestatue zu erkennen, das Fußprofil scheint abgeschlagen zu sein, aber in seinem Bereich ist das Monument im Geröll verschüttet. Die Maße sind: Höhe $>67$, Breite 59 (ohne Profil), Tiefe 175 (ohne Profil) cm, die nach unten zunehmende Größe der Buchstaben schwankt zwischen 2 bis 2,5 , der Zeilenabstand beträgt $1 \mathrm{~cm}$.

3 Einen Überblick über die Namengruppe (vgl. neuerdings etwa $R$. Bebrwald, in: F. Kolb (Hrsg.), Lykische Studien 4 [1998] 190f.) gibt L. Zgusta, Kleinasiatische Personennamen (Prag 1964) 92 ff. $\$ 97 ; 167$ ff. $\$ 355$. Danach finden sich Ermandyberis und Ermandimis (letzterer zu Unrecht bezweifelt) auch in Inedita aus Antiphellos und Andriake. Zu verwandten Namen in Limyra Wörrle, Chiron 25 (1995) 393; 406; 408, ein Paar postumer Ehrungen für einen Ermapias in Myra hat jüngst S. Şahin, EpAnat. 31 (1999) 46 f. publiziert (der Hypogymnasiarch von N. 11 ist natürlich ein Phantom).

${ }_{4} \mathrm{Ob}$ man sich hier zurecht an den in Limyra bezeugten Teviv $\alpha \sigma(\alpha \varsigma)$ und seine lykischen Entsprechungen erinnert (Wörrle, a.O. 409)?

5 Vgl. A. Balland, Fouilles de Xanthos VII. Inscriptions d'époque impériale du Létôon (Paris 1981) 153 f. mit weiteren Hinweisen.

${ }^{6}$ Zu den ägyptischen Geschwisterehen vgl. zuletzt K. Hopkins, in: P. Bonte (Hrsg.), Épouser au plus proche (Paris 1994) 79-95 (freundlicher Hinweis von D. Hennig) und N. Gonis, ZPE 133 (2000) 199 f. mit einem neuen Fall und weiterer Literatur, für Karien P. Debord, E. Varinlioğlu (Hrsg.), Les hautes terres de Carie (Bordeaux 2001) N. 16. Wie weit verbreitet das Phänomen war, ist unklar. Im Gegensatz zu Ehen von Geschwistern mit identischer Mutter scheinen solche von Geschwistern aus verschiedenen Verbindungen desselben Vaters für griechische Rechts- und Moralvorstellungen überhaupt unproblematisch gewesen zu sein. Auf wie unsicherem Boden freilich Quantifizierungsversuche stehen, haben A.-M. Vérilhac, C. Vial, Le marriage grec (Paris 1998) 82-101 gezeigt.

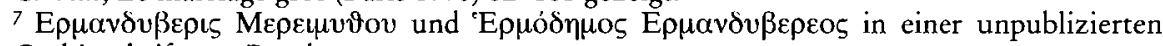
Grabinschrift von Bonda. 


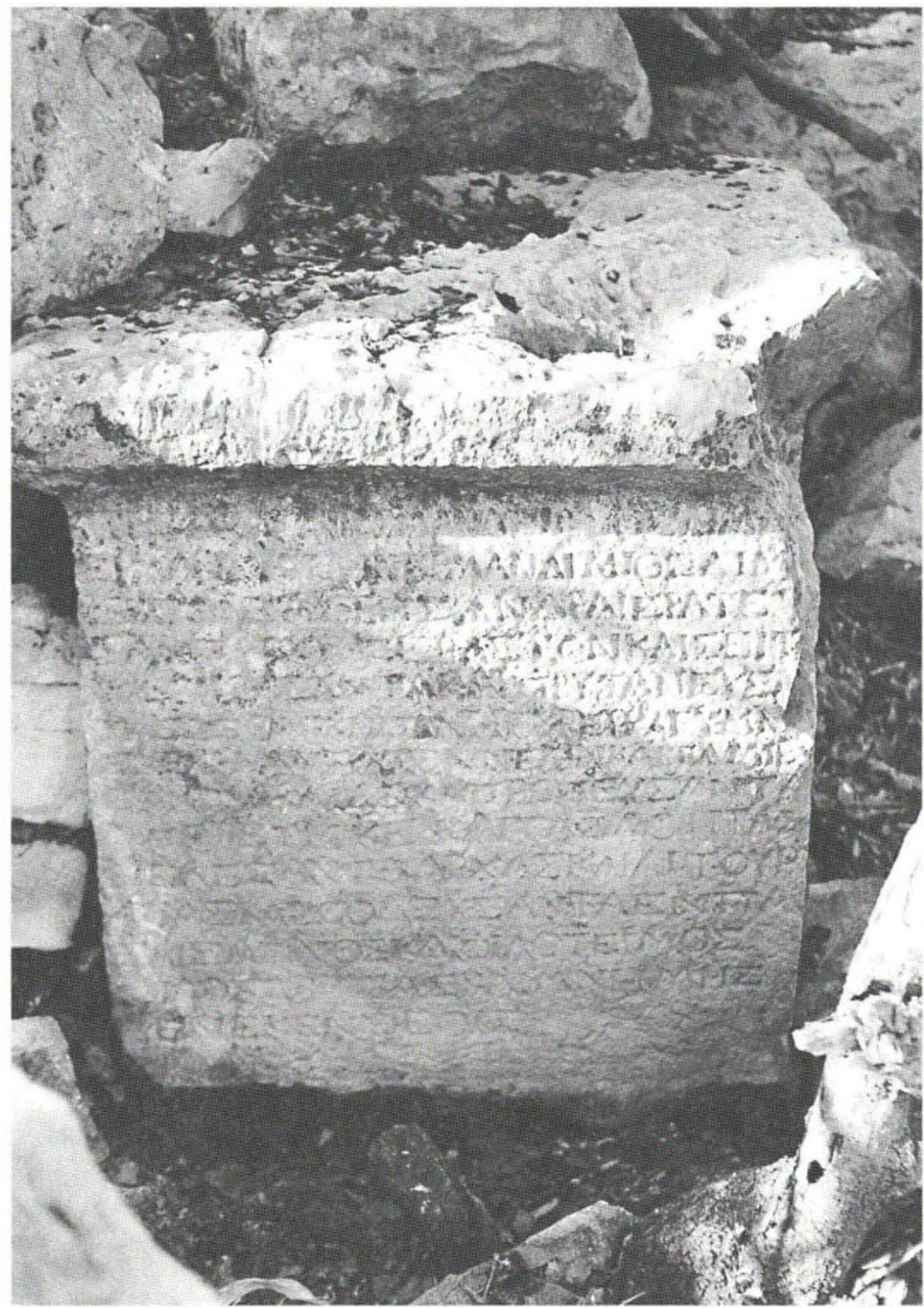

Statuenbasis des Ermandyberis 
gend zu verorten, schon weil wir ja nicht wissen, wie die anderen Familienmitglieder hießen und ob es nicht eben auch unter ihnen die uns meist ganz willkürlich erscheinende, landestypische Mischung griechischer Import- und epichorischer Traditionsnamen gab. Ein Akkulturationsgefälle zwischen Stadt und Land indiziert der onomastische Befund jedenfalls nicht.

Ermandyberis war ein politisch engagierter Patriot und hatte eine Reihe öffentlicher Funktionen in Limyra übernommen. Aus der unspezifizierten Gesamt-

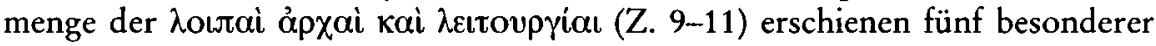
Hervorhebung wert: Zuerst ${ }^{8}$ die Mitwirkung im wohl auch in Limyra kollegial besetzten Finanzressort der $\tau \alpha$ uiol $^{9}$, danach, eine Rangstufe höher, die Leitung eines der beiden Gymnasien Limyras als Gymnasiarch der véol, der jungen Erwachsenen, der auch für den Unterricht der Epheben zuständig war ${ }^{10}$, später wieder Mitarbeit in einem Kollegium, dem der Prytanen, die wohl als Exekutivgremium für das laufende Management der limyräischen Polis insgesamt verantwort-

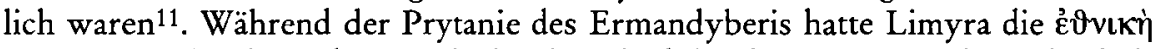

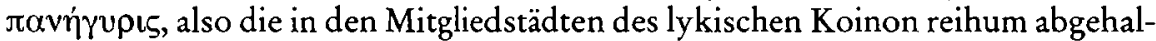
tene Bundesversammlung mit Bundesagon, auszurichten, für die amtierenden Prytanen des jeweiligen Versammlungsortes eine besondere Herausforderung und eine entsprechende Gelegenheit zum Prestigegewinn ${ }^{12}$. Danach hat Ermandyberis mit der Verteilung von Getreide an die zum Empfang berechtigte Politenelite der

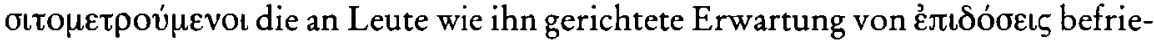
digt, was ihn eine Stange Geld gekostet haben dürfte ${ }^{13}$. Die jatpis ist Ermandyberis die Anerkennung, die er sich mit alledem verdient hatte, auch nicht schuldig geblieben und hat ihm mit dem städtischen Kaiserpriestertum die angesehenste ihrer öffentlichen Positionen anvertraut, in der er als oberster Repräsentant des Gemeinwesens bei allen kultischen und politischen Zeremonien und Staatsritualen

\footnotetext{
${ }^{8}$ Der Text gibt eine hinsichtlich Chronologie wie Bedeutung absteigende Reihung.

9 Vgl. Wörrle, Stadt und Fest im kaiserzeitlichen Kleinasien (München 1988) $114 \mathrm{f}$.

10 Wörrle, a.O. $113 \mathrm{f}$.

11 Wörrle, a.O. 109-111.

12 Ortswechsel der Bundesversammlung, anscheinend ohne festes Rotationsschema und mit Ortswahl von Fall zu Fall, bezeugt Strab. 14, 3, 3 für das vorrömische Koinon (ovvépxovta

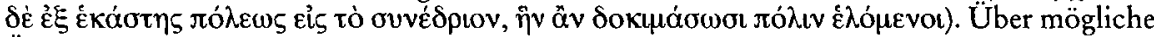
Änderungen in der Kaiserzeit lassen sich keine Aussagen machen. Bekleidung von Prytanie

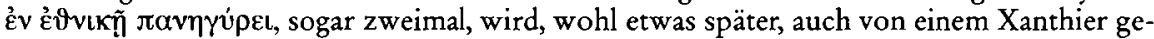
rühmt (TAM II 496, vgl. Balland, am Anm. 5 a.O. 246 ff. N. 78), und eben dort hat sich ein

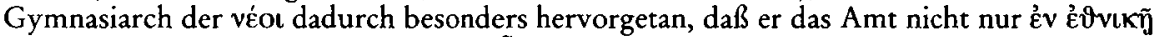

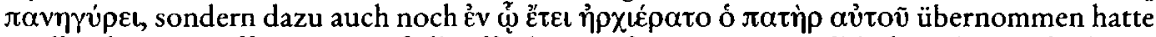
(Balland, a.O. $230 \mathrm{ff}$. N. 69). Daß die offenbar zur festgeprägten politischen Terminologie gehörende Wendung nun auch für Limyra belegt ist, widerlegt Ballands Vermutung eines speziellen Bezugs auf Xanthos und das Letoon (a.O. 233f.) und spricht für die Richtigkeit der auf Strabons Zeugnis gegründeten Vermutung auch in der Kaiserzeit fortdauernden Ortswechsels lykischer Bundesversammlungen (vgl. etwa J. Deininger, Die Provinziallandtage der römischen Kaiserzeit [München 1965] 73).

13 Wörle, a.O. 123-131; J.H.M. Strubbe, EpAnat. 13 (1989) $114 \mathrm{f}$.
} 
die erste Rolle spielte ${ }^{14}$. Wie große Pausen zwischen den einzelnen Funktionsjahren jeweils lagen, wissen wir nicht, Mindestschonfristen waren, wie in allen lykischen Städten so auch in Limyra, durch die Wahlgesetze garantiert. Die damaligen Politiker waren ja echte Amateure; Kontinuität ihres öffentlichen Engagements haben sie in aller Regel zu vermeiden getrachtet, und wenn sie sich ausnahmsweise doch zu Kontinuität gewinnen ließen, dann war das ein weiteres, besonderes Verdienst ${ }^{15}$. Unser Ermandyberis hat es sich damit erworben, daß er mehr als 25 Jahre lang $\delta \varepsilon \kappa \alpha \dot{\pi} \rho \omega \tau$ ऽ war $^{16}$, wobei die Frage direkten Anschlusses der Amtsjahre aneinander wieder offenbleibt. Viele „sabbaticals" kann er sich aber nur genehmigt haben, wenn er sehr lang aktiv war; auch das können wir nicht mehr abschätzen, aber gerade bei der Dekaprotie kam langjährige bis lebenslängliche Tätigkeit öfter vor, und den Vogel hat wohl der Mann aus Arneai abgeschossen, der das Amt mit 18 Jahren erstmals übernahm und es mit 86 immer noch bekleidete ${ }^{17}$.

Die Dekaprotoi waren aus den Buleuten, zu denen Ermandyberis mithin gehört haben muß, aufgrund besonderer Vermögensqualifikation erwählt und kontrollierten unter persönlicher Haftung für Verluste, weswegen sie eben reich sein mußten, die gesamten städtischen Einkünfte, ordentliche wie Pachten, Zölle oder Steuern und außerordentliche, hier besonders Zuflüsse aus Stiftungsvermögen. Die gesellschaftliche Einstufung solcher Leute haben uns die Inschriften abgenommen: Es sind die berühmten $\pi \rho \tilde{\omega} \tau o l ~ \tau \tilde{\eta} \varsigma ~ \pi o ́ \lambda \varepsilon \omega \varsigma$, mit deren Statuen der öffentliche Raum auch der lykischen Städte möbliert war, und alles, was ich Ihnen bisher erzählt habe, ist vielfach bezeugt: Ermandyberis war einer der kaiserzeitlichen Standard-Euergeten Lykiens. Nur: Seine Statue stand, samt Inschrift, nicht in Limyra (dort kann er natürlich eine weitere gehabt haben, aber die kennen wir nicht), sondern auf dem Bonda tepesi im, wie es scheint, durch besonders repräsentative Behausungen ausgezeichneten Ortszentrum der Kome, die heute denselben Namen trägt wie das ganze Gebiet. Sie ist dort postum durch seine schon erwähnte Frau Pteunase aufgestellt worden, und ich folgere daraus und aus dem nur dortigen Vorkommen weiterer Namensvettern, daß Ermandyberis in diesem Dorf Heimat hatte und das, was man heute seine Lebensmitte nennen würde.

In Ausübung seiner politischen Funktionen muß er aber häufig im städtischen Zentrum von Limyra geweilt haben. Der Bonda tepesi ist zerklüfteter, als man zunächst denkt. Die Wege waren zur Zeit des Ermandyberis dank Pflege, Pflasterung und Stufenanlagen sicher besser gangbar als heute, aber die Hänge waren genau so steil, im Winter regnete und gewitterte es genau so furchtbar und im Sommer hatte es dieselben $40 \mathrm{Grad}$ wie heute. Um nach Limyra zu gelangen, konnte

14 Wörrle, a.O. 101-105, vgl. Chiron 29 (1999) 355.

15 Worrle, a.O. 93-96; F. Quaß, Die Honoratiorenschicht in den Städten des griechischen Ostens (Stuttgart 1993) 334-337.

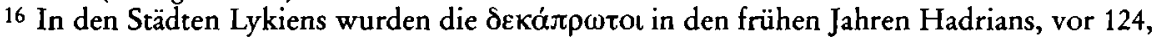

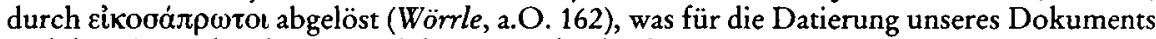
und der Biographie des Ermandyberis entscheidend ist.

$17 \mathrm{Vgl}$. Quaß, a.O. 177f. Arneai: TAM II 765. Vierzigjährige Eikosaprotie wird in einer Inschrift aus Balbura gerühmt: N.P. Milner, AS 41 (1991) 55. 
Ermandyberis entweder zu Fuß gehen oder reiten, was gute Kondition erforderte und einen strammen Tag gekostet haben muß, oder er konnte zwei Stunden sehr steil zur heute Ak liman genannten Bucht hinuntersteigen, dort auf das Linienschiff von Myra nach Limyra warten und hoffen, bei günstigem Wind und erträglicher See um das Kap von Gök burun herum bequem nach Finike zu kommen ${ }^{18}$, um von dort noch erneute zwei Stunden diesmal ebenen Weges nach Limyra zu wandern, alles zusammen auch wieder mehr oder weniger ein Tag. Wie oft oder wie selten er die Reise machte, wissen wir nicht. Bei seinen Einkommensverhältnissen wird er sich den Besitz eines Stadthauses geleistet, dort während seiner öffentlichen Aktivitäten residiert und auch seine Kinder für die Zeiten des Gymnasionbesuches standesgemäß untergebracht haben; auch davon wissen wir nichts. Um von dem möglichen Maß an Bequemlichkeit und vielleicht sogar Luxus, das er sich in Bonda leistete, eine konkrete Vorstellung zu bekommen, müßte man vom Survey zur Ausgrabung seines Hauses fortschreiten, woran gar nicht zu denken ist. Die erfrischende Dusche, die wir auf dem Bonda tepesi vermißten, hatte er jedenfalls auch nicht, Wasser kam ausschließlich aus der Zisterne, aber das hat ihn bei ganz anderen zivilisatorischen und hygienischen Standards als heute wohl wenig gestört, und er hatte es ja im Gegensatz zu uns auch von klein auf gelernt, damit umzugehen. Wie es um seine $\pi a \iota \delta \varepsilon i \alpha$ stand, können wir gar nicht einschät-

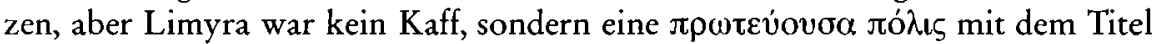

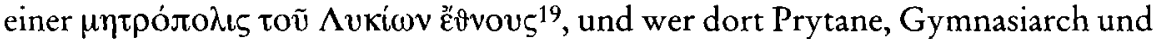
Kaiserpriester war, mußte sich schon einigermaßen souverän auf der kulturellen Höhe der traianisch-hadrianischen Zeit bewegen können.

Das Tätigkeitsbild eines Dekaprotos können wir trotz vieler, aber eben monotoner epigraphischer Belege für die Funktion nur in rudimentären Ansätzen konkretisieren. Immerhin war unter den römischen Rechtsgelehrten wenigstens der späteste, Modestin, zu der Ansicht gekommen, daß so jemand nicht nur das patrimonii onus trug, über das anscheinend Konsens bestand, sondern auch ein corporale ministerium erbrachte ${ }^{20}$. Das den Dekaprotoi gesetzte Leistungsziel scheint im Prinzip immer dasselbe, der Eingang vorher als Pauschalsummen festgelegter Geldbeträge in die Stadtkasse, gewesen zu sein. Da diese Beträge als Ausgaben auch schon wieder vorweg verplant waren, mußten die Einnahmen zuverlässig und pünktlich kommen, notfalls aus dem eigenen Vermögen der Dekaprotoi, de-

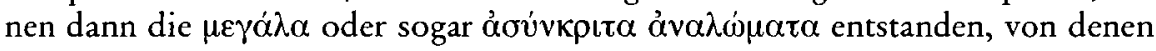

18 OGI 572 (vgl. L. Robert, Noms indigènes dans l'Asie-Mineure gréco-romaine [Paris 1963] $35 \mathrm{f}$. mit Taf. II) enthält ein wohl in das 2. Jahrhundert $\mathbf{n}$. Chr. zu datierendes Dekret von Myra über die als städtisches Monopol vergebene Linienschiffahrt nach Limyra. Als Halteplätze waren darin außer Andriake die Mündung des Ölü deniz am Westfuß des Bonda tepesi

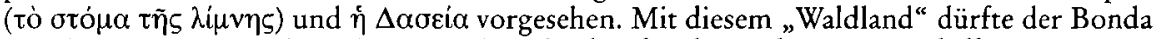
tepesi insgesamt gemeint sein. Von seinen beiden für das Anlegen von Schiffen geeigneten Buchten kommt zur Nutzung von der Siedlung von Bonda aus nur die westlichere, eben der Ak liman, in Frage.

19 Wörrle, a.O. 240 mit dem Hinweis auf Balland, a.O. $176 \mathrm{f}$.

20 Dig. 50, 4, 18, 26. 
eine Inschrift aus Idebessos spricht ${ }^{21}$. Das war aber der, dann natürlich spektakuläre, Ausnahmefall, in dem das System versagt hatte. Von der Normalität seines Funktionierens zeugt dagegen eine Inschrift der späten 130er Jahre aus der zu Kadyanda gehörigen Kome von Dereköy im äußersten Nordwesten Lykiens ${ }^{22}$, nach der es für die zehnprozentige Steuer auf den Ertrag des Getreideanbaus, die бıтıкं $\delta \varepsilon \kappa o ́ t \eta \eta$, eine dorfbezogene Pauschale gab, die sich bei der jährlichen Abrechnung mit dem Dekaprotos auch als übererfüllt erweisen konnte; es entstanden dann Überschüsse, die dem Dorf für die Finanzierung von Gemeinschaftsaufgaben verblieben. Die Komenpauschale war die Zusammenrechnung der Einzelveranlagungen aller Flurstücke des Dorfgebietes. Ihr Besitz war, auch dies alles läßt die erwähnte Inschrift von Dereköy erkennen, nicht zeitlos stabil, sondern ständigem Wechsel durch Erbgänge und Immobiliengeschäfte unterworfen und überhaupt bunt gestreut, wobei die Eigentümer, die auch Personengemeinschaften mit verschieden gestückelten Anteilen sein konnten, keineswegs alle ortsansässig waren. Da nach dem Anteil am Gemeindegebiet nicht nur die besonders wichtige oıtıkn

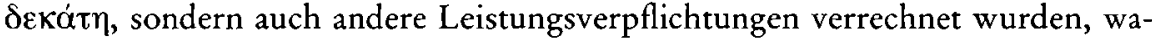
ren die Besitzverhältnisse ständig sorgfältig zu kontrollieren, was dadurch ermöglicht wurde, daß einzelne Dekaprotoi jeweils für bestimmte Dörfer zuständig waren, und natürlich waren auch die Abgaben in der gerechten Höhe umzulegen und einzutreiben, wobei uns die sicher besonders sensiblen Problemkreise von Vermarktung und Adaerierung der Ernten gar nicht zugänglich sind, waren schließlich den einzelnen Leistungspflichtigen und der Gesamtgemeinde korrekte Quittungen auszustellen. Aus Ägypten haben wir eine ganze Reihe von Papyri, die die Bedeutung dieser Quittungen einschärfen, auch Beschwerden über angeblichen Quittungsbetrug und ungerechtfertigte Zwangsmaßnahmen von Dekaprotoi dokumentieren, und in Kleinasien gibt es natürlich die Ehreninschriften, die einzel-

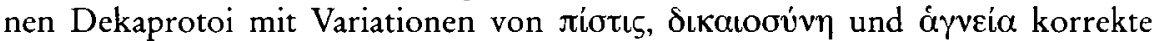
Amtsführung attestieren. Daß sie die Ausnahme, Korruption und Ausbeutung die Regel gewesen seien, folgt daraus nicht so selbstverständlich, wie man es häufig liest, und die gerade für Dekaprotoi typischen langen Amtszeiten, von denen ich vorhin gesprochen habe, sind wohl nicht nur die Konsequenz aus der Vertrautheit mit den örtlichen Verhältnissen, die für eine effiziente Amtsführung nötig war, sondern auch ein Indiz für eine Art Vertrauensverhältnis zwischen den Dekaprotoi und „ihren“ Dörfern, die langfristige Stabilität wünschenswert erscheinen ließ.

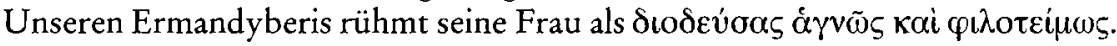
Das ist ganz originell formuliert und, wie immer, wenn keine unmittelbaren Parallelen zur Hand sind ${ }^{23}$, mit entsprechenden Vorbehalten zu interpretieren, aber

21 TAM II 838.

22 Wörrle, Chiron 27 (1997) 399ff., zum Wirken der zuständigen Dekaprotoi 447-458, auch mit den für die folgende Skizze nötigen Nachweisen.

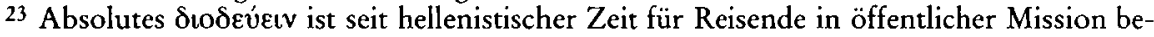
zeugt. So bezieht sich etwa Antiochos III. bei dem von Ptolemaios in seinen syrischen Dör-

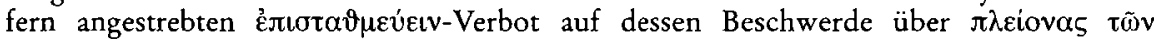

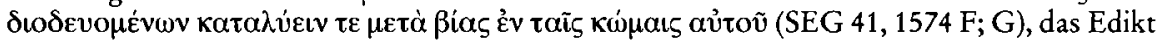


es scheint doch gemeint zu sein, daß er als professioneller Wanderer viel im Außendienst zu tun hatte und dabei gerecht, sogar großzügig war. Wir haben dann ein bißchen hinzuzulernen: Ermandyberis war kein abgehobener, fremder Honoratior, der ab und zu aus der fernen Stadt in den Dörfern des Bonda tepesi auftauchte, um Steuern zu kassieren, und, wenn er Pech hatte, von den Bauern gesteinigt wurde, wie Libanios die Sache für das Antiocheia des 4. Jahrhunderts hinstellt ${ }^{24}$, sondern ein Mann vom Land, ein muhtar-Typ - aber vermutlich kein engstirniger - aus dem Dorf, der dort alle kannte und von allen geachtet war und auf dieser sozialen Basis in seinem Bonda und anderen Dörfern auf dem Bonda Tepesi mit ihren wohl nicht anders als in der Kome von Dereköy klein strukturierten Besitzverhältnissen die übergeordneten Interessen der Polis nicht nur bürokratisch korrekt verwalten, sondern auch in persönlicher Glaubwürdigkeit und Nähe mit Autorität und Augenmaß vertreten konnte. Es ist dies eine Facette von StadtLand-Beziehung, die sich uns in seiner Person erstmals deutlich artikuliert, aber wir haben keinen Grund zu glauben, daß Ermandyberis ein Einzelfall unter den vielen Dekaprotoi war, von denen wir wissen, und die schon deshalb nicht die institutionalisierten Ausbeuter der Landbevölkerung sein konnten, weil sie genau so ja auch für die Abgaben zuständig waren, die im städtischen Siedlungszentrum und, soweit vorhanden, im Hafen anfielen ${ }^{25}$, und weil sie selbst ja das Fungieren von Dekaprotoi durchaus von beiden Seiten zu sehen bekommen konnten wie jener Ermadortas, der in der Grundbesitzerliste von Dereköy auftaucht, dort mithin Steuern zahlen mußte, und am selben Ort auch als Dekaprotos gewirkt, also Steuern kassiert, und einen Überschuß hinterlassen hat ${ }^{26}$. In seinem Fall wissen wir nur nicht, ob die Kome von Dereköy auch seine Heimat war; er gehört dort nämlich nicht zu den großen Bauern und könnte noch andernorts begütert gewesen sein.

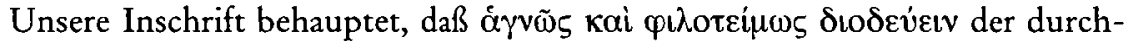

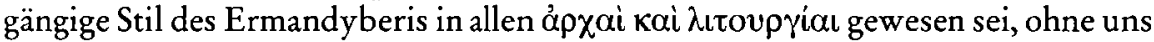
diese Funktionen unterhalb der Dekaprotie im einzelnen zu verraten. Man denkt

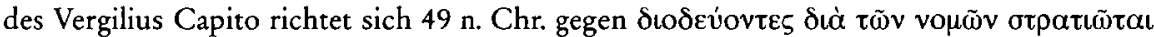

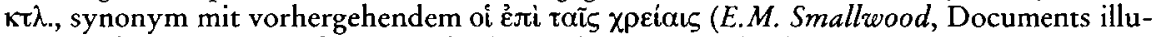
strating the principates of Gaius, Claudius and Nero [Cambridge 1967] 582). Ganz entspre-

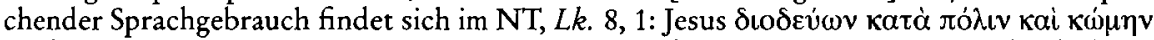

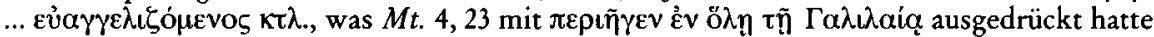

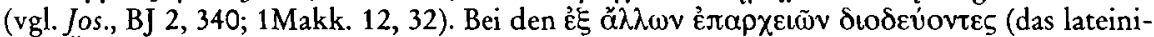
sche Äquivalent ist commeare) hat das Edikt des Sotidius Strabo (St. Mitchell, JRS 66 [1976] 107 f.) wie OGI 613 eher bloßes Durchreisen im Auge, aber eine klare Grenze läßt sich hier naturgemäß kaum ziehen.

24 Or. 47, 7, vgl.J.H.G. Liebeschuetz, Antioch (Oxford 1972) 202.

${ }^{25}$ Leider ist die, Weitergabe von Zolleinnahmen betreffende Inschrift aus Myra, die ich in J. Borchbardt (Hrsg.), Myra (Berlin 1975) 286-300 veröffentlicht habe, so zerstört, daß hinsichtlich der Rolle von Deka-/Eikosaprotoi S. 290 f. über Vermutungen nicht hinauszukommen war; kein Fortschritt bei H. Schwarz, EpAnat. 33 (2001) 15-37.

26 Am Anm. 22 a.O. B I 16; II $57 \mathrm{ff}$. 


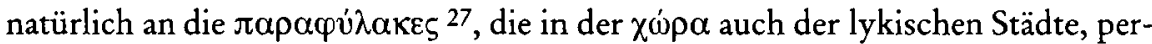
sönlich präsent und nicht von irgendeinem Schreibtisch aus, den Polizeidienst leiteten, und da wir auch diese Funktion vorzugsweise aus den Ämterlisten von städtischen Ehrenstatuenbasen kennen, hat sich hierüber ein Klischee festgefressen, das ich am besten mit den Worten von Keith Hopwood - sie sind seine Summe einer Studie über die Verhältnisse in Kilikien - formuliere ${ }^{28}$ : „The divorce between police and policed was total: the latter were the partially-hellenized native populations living on the fringes of the city-state; the former were the cultured and hellenized governing classes who imposed an alien language and laws on their inferiors." Keinen geringen Beitrag zu diesem Bild hat eine Inschrift aus dem Territorium von Hierapolis, dem heutigen Pamukkale, geleistet ${ }^{29}$. Sie begrenzt die Ansprüche der dortigen $\pi \alpha \rho \alpha \varphi \dot{\lambda} \lambda \alpha \kappa \varepsilon \zeta$ an die Dörfer, in denen sie dienstliche Station machten (die $\dot{\varepsilon} \pi \iota \delta \eta \mu i \alpha$, von denen die Rede ist, scheinen mir nur ein anderer

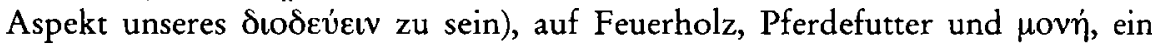
Dach über dem Kopf. Requirieren sie mehr, drohen Konfiskation und Strafe. Ehrengeschenke an die $\pi \alpha \rho \alpha \varphi v ่ \lambda \alpha \kappa \varepsilon \varsigma$ scheinen üblich gewesen und z.T. erpreßt worden zu sein; sie bleiben erlaubt, aber der freie Wille der Dörfer wird ausdrücklich geschützt. Diese Inschrift gäbe es nicht, wenn keine Mißstände abzustellen gewesen wären, aber man darf nicht überlesen, daß es sich um eine Dekretinitiative der ßov $\lambda \eta$ von Hierapolis handelt, mit der sich die Honoratioren eines „citystate“ massive Beschränkungen zugunsten von dessen „populations on the fringes" und im Interesse einer Stadt und $\chi \omega \dot{\rho} \alpha$ vereinigenden, rechtsstaatlichen Poliskonzeption auferlegten - und es steht ihrem negativen Bild dörflicher Polizeierfahrung ausgleichend die Dankesinschrift eines Dorfes von Prusa am Olymp gegenüber ${ }^{30}$, dem ein korrekter $\pi \alpha \rho \alpha \varphi v ́ \lambda \alpha x$ zum Beschützer geworden war. Die ,rural chiefs', denen ich begegnet bin, schienen mir überall in der Regel harte Leute zu sein, und nicht jeder, der an Pteunases Statue vorbeigekommen ist, muß nur gute Erinnerungen an Ermandyberis gehabt haben; "total divorce" ist aber wohl kaum die geeignete Kategorie, seine Beziehungen zu den Menschen auf dem Bonda tepesi zu charakterisieren, mit großer Wahrscheinlichkeit das Gegenteil, aber wir haben eben bisher die Möglichkeit, daß auch ein $\pi \alpha \rho \alpha \varphi \dot{\lambda} \lambda \alpha \xi$ aus dem Land, für dessen Ordnung er sorgte, selbst kommen konnte, mit Ausnahme von Christof Schuler ${ }^{31}$, gar nicht ins Auge gefaßt.

Schneller als Ermandyberis, in etwa vier Stunden, war Hieratikos, Sohn des Trebemis, in Limyra ${ }^{32}$. Er war zu Hause im Peripolion von Asarönü und wird in

27 Am Anm. 9 a.O. 115; 149f.; Th. Corsten im Kommentar zu I.Prusa ad Olympum (Bonn 1991) 23; St. Mitchell, Anatolia I (Oxford 1993) 195-197.

${ }^{28}$ In: Mitchell (Hrsg.), Armies and Frontiers in Roman and Byzantine Anatolia (1983) 180.

29 OGI 527, vgl. L. Robert, Etudes anatoliennes (Paris 1937) 103-105.

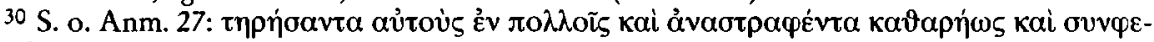

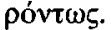

${ }^{31}$ Ländliche Siedlungen und Gemeinden im hellenistischen und römischen Kleinasien (München 1998) 277.

32 Die Inschrift, auf der das Folgende beruht, habe ich Chiron 29 (1999) 353-370 veröffentlicht. 
der Inschrift einer dort errichteten Statuenbasis für seine verstorbene Frau als $\pi \rho \omega \tau \varepsilon v \dot{\omega} \omega v$ dieser Siedlung bezeichnet, an deren Rechtsstellung hier nur soviel interessiert, daß auch sie als ländliches Subzentrum Komenstatus hatte. Hieratikos und seine Frau hatten mit dem gemeinsam bekleideten Kaiserpriestertum gegen Ende des 2. Jahrhunderts n. Chr. ebenfalls den Weg an die Spitze der städtischen Ämterhierarchie in Limyra geschafft, gewiß nicht ohne die üblichen Vorleistungen kontinuierlichen öffentlichen Engagements, wie wir sie bei Ermandyberis studieren konnten. Während dessen Statue anscheinend ein privates Unternehmen seiner Frau Pteunase war, wird bei der Kaiserpriesterin in Asarönü ausdrücklich auf das Dekret von Rat und Volk von Limyra hingewiesen, das die Errichtung ihres Denkmals durch ihre pietätvollen Söhne autorisierte und zugleich nobilitierte. Fragen nach Mentalitäten sind notorisch besonders heikel, aber es wäre doch interessant, eine Ahnung davon zu haben, was ,Stadt in den Augen derer bedeutete, die mit Ermandyberis und Hieratikos auf dem Land wohnten. Wäre die Beziehung mit negativen Sentiments überfrachtet gewesen, hätten Ermandyberis und Hieratikos nicht gerade im dörflichen Ambiente sich ganz ausschließlich und demonstrativ mit Erfolgen vorgestellt, die nicht nur für uns, sondern gewiß auch für ihre Zeitgenossen mit dem städtischen Zentrum der Polis verbunden waren und auf dort geleisteten Funktionen beruhten.

Im Vergleich zu Limyra war das nördlich angrenzende Arykanda, so imposant es sich heute dank guter Erhaltung und jahrelanger Ausgrabungen dem Besucher präsentiert ${ }^{33}$, eine bescheidene Gebirgs-Polis, und Hieron, der Bürgermeister ( $\left.\delta \eta_{\mu} \alpha \rho \chi \varsigma \varsigma\right)$ der etwa drei Wegstunden entfernten Kome von Kilepe an der Grenze zu Arneai hatte es selbst in den bescheidenen Verhältnissen von Arykanda nicht so weit gebracht wie die beiden Herren aus der $\chi \omega \dot{\omega} \alpha$ von Limyra: Neben dem Demarchenamt im heimatlichen Dorf war er Priester, wenn auch nur des Zeus und

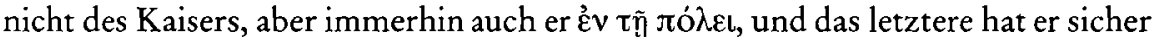
nicht aus topographischer Pedanterie, sondern wegen des höheren Wertes herausgestellt, den sein Priestertum eben deswegen hatte, weil es eines in der Stadt war ${ }^{34}$. Noch ein wenig deutlicher tritt dieser Aufsteigerstolz in den Inschriften zweier ebenfalls schon von Christof Schuler ${ }^{35}$ bemerkter Sarkophage in der zu Perge gehörigen Lyrboton Kome am Westrand Pamphyliens hervor: Das entsprechende Vermögen hatte wohl schon der Vater zusammengebracht, für soziale Mobilität hat es der Sohn genutzt, dem der zugleich trauernde und beglückte Vater aufs

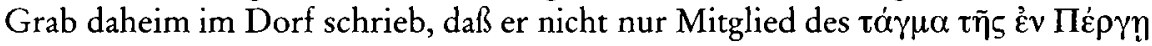
үepovoías geworden sei, sondern von dem honorigen Klub mit einem lebenslangen Priestertum ausgezeichnet wurde und dafür 600 Denare springen ließ. Understatement ist das nicht, und Neider mag es im Dorf gegeben haben. Das ändert nichts daran, daß die Stadt die Standards vorgab und darstellte, nach denen sich

${ }^{33}$ Vgl. die Beiträge von C. Bayburtluoğlu und P. Knoblauch zu: J. Borchbardt, G. Dobesch (Hrsg.), Akten des II. Internationalen Lykiensymposions (Wien 1993) II 119-130 sowie $P$. Knoblauch, Cb. Witschel, ArchAnz. (1993) 229-262.

${ }_{34} \mathrm{Zu}$ Hieron vgl. Wörrle, in: Fremde Zeiten. Festschrift J. Borchhardt (Wien 1996) 153-160.

35 S. Şabin, EpAnat. 11 (1988) 162f. N. 158f. mit den Überlegungen Schulers, a.O. 280 f. 
auch in diesem Fall die Wertvorstellungen der Leute auf dem Land ausrichteten. Partizipation daran muß für alle erstrebenswert gewesen sein, vor allem bei den großen Festen in der Stadt Zugehörigkeit zur großen Polis zu erleben, auch wenn man im Dorf dafür vorher die Rinder züchten mußte, deren Hergabe zum gemeinsamen Opfer und anschließenden Schmaus der Gemeinde und ihrer Gäste sicher ein Opfer war ${ }^{36}$.

Die Leute vom Land mit Stadtconnections, die wir betrachtet haben, sind zu ihrem sozialen Rang mit Geld gekommen, über das sie auf Grund von Vermögen verfügten, die im Rahmen ihrer Heimatdörfer außergewöhnlich groß waren. Worauf die wirtschaftliche Blüte des kaiserzeitlichen Bonda tepesi beruhte, hat Andreas Konecny in seinen beiden Aufsätzen über die Ölpressen gezeigt, die dort oben bis in die Dörfer an der Vegetationsgrenze für Olivenbäume ein ubiquitäres Phänomen $\operatorname{sind}^{37}$. Diese Ölproduktion muß intensiv, markt- und wohl sogar exportorientiert, in Bonda selbst die überwiegende Kultur gewesen sein, und wenn, was wir nicht ausschließen können, Ermandyberis nicht noch anderswo in Land oder gar in Spekulationsgeschäfte investiert hatte, beruhte sein Reichtum auf Olivenplantagen, die er um Bonda herum besaß, wobei man freilich nicht an afrikanische oder hispanische Dimensionen denken darf, daneben vielleicht auf Herden von Ziegen, die man im Gegensatz zu den Oliven noch heute auf dem Bonda Tepesi trifft und damals in Limyra-Stadt nach dem Ausweis der Knochenanalysen ${ }^{38}$ anscheinend mehr als alles andere Fleisch verspeiste. Mustafa Adak und Orhan Atvur haben das Grabhaus einer Familie in der ostlykischen Hafenstadt Olympos publiziert, in der es um die Wende vom 2. zum 3. Jahrhundert n. Ch. der Onkel Eudemos als Reeder im Überseegeschäft mit dem Pontos zu dem großen Geld gebracht hatte, von dem dann der Neffe Zosimas sicher nur einen kleinen Teil in ein aufwendiges Familienmonument investierte 39 . Auf die Frage, was Eudemos aus Lykien nach dem fernen Norden verschifft haben könnte, sind Adaks Antwor$\operatorname{ten}^{40}$ nicht recht überzeugend. Das Öl, natürlich nicht nur das vom Bonda Tepesi, wäre ein solcher Exportartikel, mit dem nicht nur Händler, sondern auch Produzenten wie unser Ermandyberis reich werden konnten, so reich, daß ihnen der Sprung in die städtische, society ' gelang. Zu Rentiers, absentees, städtischen Honoratioren oder wie immer man die falschen, mindestens einseitigen Bilder benennen will, sind Leute wie er dadurch nicht geworden.

So zu reden, als ob es eine Geschichte dieses Mannes gäbe, war leider nur ein Spiel, das sich am Ende in dem Nebel verliert, der oft auch im schönsten Sommer auf dem Bonda Tepesi einfallen kann. Die Struktur, auf der ich es zu entfalten ver-

36 Für das Demostheneia-Fest im hadrianischen Oinoanda waren 27 Rinder vorgesehen, deren Lieferung durch die Funktionsträger (13) und die Dörfer (14) der Polis das Gründungsdokument regelte (am Anm. 9 a.O. Z. 68-85).

37 ÖJh. 67 (1998) B 121-194; 68 (1999) B 141-170. Vgl. Marksteiners (o. S. 286 f.) Erwägungen zu möglichen Facetten weiterer landwirtschaftlicher Aktivität.

38 G. Forstenpointner, G. Gaggl, ÖJh. 66 (1997) 419-426.

39 EpAnat. 28 (1997) 11-31.

40 A.O. 24. 
sucht habe, ist die Annahme einer recht engen interaktiven Verzahnung von Stadt und Land und von unverkrampfter Akzeptanz einer administrativen Ordnung, die im Normalfall so gut funktionierte, daß die Polis aus der Sicht ihrer zentralen wie ihrer ländlich-marginalen, aber eben keineswegs von naiven Eingeborenen besiedelten Komponenten als gemeinsamer Wert empfunden und als gemeinsame Aufgabe von beiden Seiten auch gewollt wurde ${ }^{41}$. Der Weg in den historischen Alltag von Dorf und Stadt ist immer noch weit, und über das Lykien der hohen Kaiserzeit hinaus beansprucht mein Experiment keine Geltung.

41 In den pessimistischen Rahmen von Dialogverweigerung, unüberbrückbarer zivilisatorischer Distanz, Ausbeutung und Vergewaltigung, den Mitchell am Ende seiner Synthese über das ländliche Anatolien zeichnet (am Anm. 27 a.O. 165-197), passen unsere Beobachtungen in gar keiner Weise. Die Lehre daraus darf nicht die Leugnung der Schattenseiten (vgl. dazu besonders noch P. Herrmann, Hilferufe aus römischen Provinzen [Göttingen 1990]), muß vielmehr Zurückhaltung beim Entwurf verallgemeinernder Gesamtbilder sein, von denen wir das jüngste $J$. Nollé, in: W. Eck (Hrsg.), Lokale Autonomie und römische Ordnungsmacht in den kaiserzeitlichen Provinzen vom 1. bis 3. Jahrhundert (München 1999) 93-113 verdanken. Mit seiner reichen Dokumentation eröffnet es auch den Zugang zur einschlägigen Vorgängerforschung. 